\title{
An In-Depth Study of Multimedia Elements for Programme Production in Television Stations in Lagos State, Nigeria
}

\author{
Benjamin Eni-itan F. Afolabi \\ Department of Mass Communication, Achievers University, P.M.B 1030, Owo, Ondo State, Nigeria
}

\begin{abstract}
Multimedia refers to access of information by a variety of display media elements: text, graphics (vector), images (bitmap), audio (sound), motion pictures (animation) and video. They are weaved into a computer based presentation or TV programmes. Multimedia graphics has played a significant role in design and visual communication; while television is a medium for accessing those designs for disseminating information. Multimedia elements are experienced in day to day activities and also have a critical impact in television programming. This paper therefore aims to examine multimedia elements and its features for the production of programmes in television stations. The area of study is Lagos State, Nigeria. A concise survey was carried out in the study area which uncovers the problems of multimedia production for television; hence, adequate measures to resolve the challenges were proposed.
\end{abstract}

Keywords: Multimedia Content, Media Elements, Linear Mode, Programmes, Visuals

DOI: $10.7176 / \mathrm{ADS} / 71-05$

Publication date:March $31^{\text {st }} 2019$

\section{Introduction}

Technological evolution for many centuries has generally influenced the mode of hearing, feeling, seeing and perception. This evolution is a gradual development from simple to a more complex or better form; which brings about innovation. Innovation is part of everyday lives redefining the world. Multimedia is an innovation to shape the culture of communication and direct senses, be it consciously or sub-consciously for specific purpose (Fowler 2000). Communication, if not interpersonal would be through mass media. Part of the innovation in the recent time is new media: interactive TV and a webcast (streaming via the internet- a virtual network that involves many people sharing information at a time). It will definitely be less effective without the use of Multimedia elements meant for visual communication (Afolabi 2016).

'Visuals' in communication explicitly point to the media elements while incorporating aural capabilities. In same way, Television as a product of technology is equally an audio-visual platform that appeals to the sense of sight and ear. It is used to inform and be informed, entertain and be entertained. Interestingly, Television and other means of communication have opened doors for instantaneous accessibility to information. But as observed by Nayyar (2007), despite this unique breakthrough, man's quest for communication and information is still noticeably high hence the increasing need for multimedia technology. It will be sane to clarify that multimedia is a subset of new media; and also the integration of more than one element either text, images, audio, video and animation to communicate meaningfully. This is what television programming entails; it is not only about the box neither is it a one man show. What is experienced on television is a combination of the media elements. Text is either used for subtitle, teletext or needed as on-screen message in terms of montages and credits. Images are needed in all ramifications since the medium of television is strictly 'visual'. Videos are the core of television programming, recorded visuals -rushes, are different from still images, and when life is put into it (still images), such becomes animation i.e. motion pictures. The creative blend of the above-mentioned media elements creates a perfect story. Also, all the multimedia elements can be manipulated to achieve a stunning special effect for production. Individually, an element's effect is reduced and cannot make much impact compared to hybrid form; but in composition, they aid a better multi-sensory experience (Afolabi 2016). The power of multimedia cannot be under estimated because even right from time, humans, when it comes to communication related orally and pictorially (Nayyar 2007) - a multi-channel of communication that its resultant effect surpasses the lone agelong written language. 


\section{Television \& Multimedia Elements: Text, Graphics, Images, Sound, Video \& Animation}

Television is an electronic means of communication; major attribute is the encoding, distribution and decoding of multimedia contents via electronic technology. Television cannot function to the level of influencing the viewers without multimedia contents that comprise: Text, Images, Sound, Video and Animation which are also referred to as media elements. In practice, according to Campbell, Martin and Fabos (2012), television is solely an industry of producers and networks that sell audience of television to the advertisers - the adjuncts; in which, audience measurement in return, helps the advertisers to detect the type of viewers and population patronising a particular content. But the fact and question is what actually are the viewers watching if not the multimedia contents built with media elements. On this note it is pertinent to take a close look at the multimedia elements.

\subsection{Text}

The print media is the oldest and first well known mass medium; books and magazines respectively that use text (Daramola 2003). It is not only the print media that communicates with the use of letters; Television, as opined by Akpan (1990), the greatest communications mechanism ever designed and operated by man, likewise, employs texts to bring visual message to reality. Vaughan (2011) wrote that the first communicative inscriptions (Figure 1) were made on mud tablets in the Mediterranean Fertile Crescent; this could be traced back to six thousand years ago. History has it in record that the rank and file did not have access to the cuneiforms except the ruling classes and the priests. However, that was then but in twenty-first century, though information is accessible by virtually all and sundry but texts and ability to decode it empowers (Vaughan 2011).

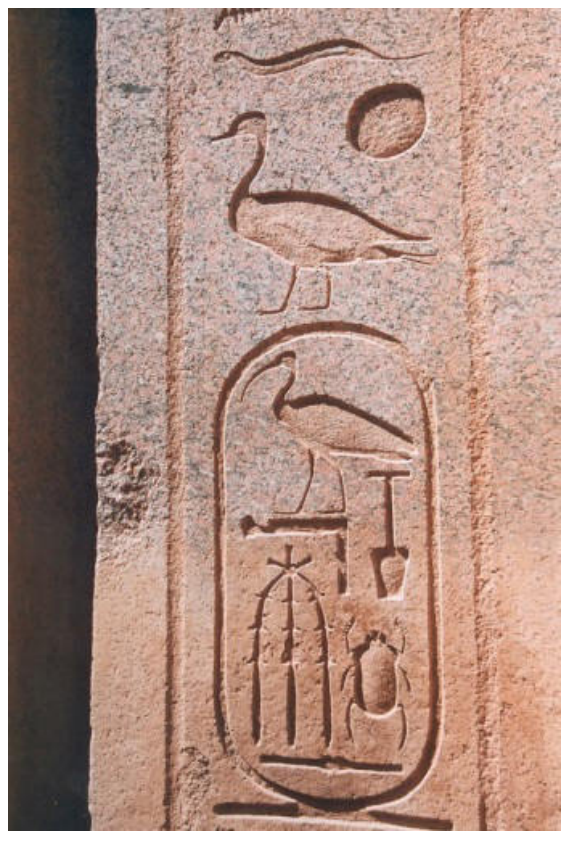

Fig. 1, Shenu: Ancient Egyptian Inscription-

Hieroglyphs on mud surface; enclosed texts depict a royal name. (Source: Gérard Ducher, 2006)

Creative Commons; Attribution-Share Alike 2.5 Generic License

(https://creativecommons.org/licenses/by-sa/2.5/deed.en)

Text is readable on any medium because of the coding system involved known as Character set and Alphabets: ASCII (American Standard Code for Information Interchange), Extended Character Set and the UNICODE (Afolabi 2016). Vaughan (2011) ascertained that ASCII is a 7-bit character coding system that depicts a letter or symbol of English alphabets; owing to this technology, monitors (the screen) can easily display characters. The Extended Character Set is filled with ANSI (American National Standard Institute) standard characters which often use symbols. ANSI is applicable to Hyper Text Mark-up Languages, an essential tool for authoring webpages. Meanwhile, in contrast, Vaughan (2011) further maintained that UNICODE is 16 bit architecture for international language alphabets and character encoding; it supports multi-lingual text of the world. Text as a form of multimedia element and means of communication brings about typography, the design and use of 
typefaces to enhance visual message. A font is a group of one or more types, bound by common characteristics in appearance that sets them apart from other fonts. Typeface on the other hand, refers to the family of types: Arial, Gothic, Tiresias et cetera with all their attributes such as narrow, regular, bold, rounded etc. (Gibson 1979).

Typically, a type will either be a PostScript, TrueType or OpenType. PostScript, invented by Adobe is a 'type one' font comprising the outline and the metric font. Both features are needed for detailed output; precisely, quality printing production of the font (Braha and Byrne 2011). TrueType and OpenType are the products of the joint efforts of Microsoft, Apple and Adobe. TrueType makes use of only a file for it to be displayed on the screen and for printing while OpenType supports Extended Character Set, it also excels in its ability to be used on multiple platforms: Windows and Mac alike. Specifically, Adobe paired with Microsoft to invent OpenType while Apple teamed up with Microsoft for the actualisation of TrueType (Braha and Byrne 2011). Legibility, Quinn (2005) stressed is crucial in typography for readability and communication but what is the essence of legibility without effective spacing. 'Kerning', 'tracking' and 'leading' (Figure 2) appropriately will not only enhance legibility, it will also create an excellent visual appeal for both screen and print. As a result, spacing must be considered for legibility; it is then readability can be achieved for sensible communication. At the same time, the striking essence of legibility (to make reading possible) necessitated the legibility index chart designed by the United States Sign Council (See Table 1 and 2).

Table 1: United States Sign Council (USSC) Legibility Index for Font Size and Distance

\begin{tabular}{|c|c|c|c|c|}
\hline Letter Style & Letter Colour & $\begin{array}{l}\text { Background } \\
\text { Colour }\end{array}$ & Legibility & Index \\
\hline \multirow[b]{2}{*}{ Helvetica } & \multirow[b]{2}{*}{ Black } & & Uppercase \& Lowercase & All Caps \\
\hline & & & 29 & 25 \\
\hline Helvetica & Yellow & & 26 & 22 \\
\hline Helvetica & White & & 26 & 22 \\
\hline Clarendon & Black & & 28 & 24 \\
\hline Clarendon & Yellow & & 31 & 26 \\
\hline Clarendon & White & & 24 & 20 \\
\hline
\end{tabular}

Source: (Braha and Byrne 2011)

Description: For the sake of legibility, texts are known to possess ascenders and descenders (Figure 2) common with Sentence Case, due to this, some words are more conspicuous than others. Thus it causes the difference (as reflected on table 1) when using letters of 'Uppercase and Lowercase' and 'All Capital letters'. A live example (in line with Table 1) is using white Clarendon letters on a background that is black and its capital letters are fixed for 1 inch, with this setting; it should be readable right from $24 \mathrm{ft}$ (uppercase and lowercase) or $20 \mathrm{ft}$ (all capitals). Inferably, if the character is set to 10 inches, it will be seen at a distance from $240 \mathrm{ft}$ and 200 $\mathrm{ft}$ - 'uppercase and lowercase' or 'all capitals' respectively.

Table 2: Adaptation of United States Sign Council (USSC) Legibility Index for different Screens

\begin{tabular}{|c|c|c|}
\hline Format & Frame Size & $\begin{array}{c}\text { Point Size } \\
\text { (Equivalent to 1 Inch Height \& } \\
\text { Legible from 30 Feet Away) }\end{array}$ \\
\hline Film 4K & $2048 \times 1556$ & 208 pts. \\
\hline Film 2K & $4096 \times 3112$ & 104 pts. \\
\hline HD/ HDTV 720p & $1280 \times 720$ & 65 pts. \\
\hline DV NTSC & $720 \times 480$ & 35 pts. \\
\hline
\end{tabular}

Source: (Braha and Byrne 2011) 
Typefaces can be categorised mainly into serif and san serif. Serif fonts are letters with short strokes at the top and bottom while San-Serif fonts are without the ornamental lines (serif). Vaughan (2011) revealed that 'Sans' is a French word meaning 'without'. Additionally, UrbanFonts (2016) illustrated that San Serifs are suitable for the screen while Serifs are the best for Print media. It explained that Serifs are meant for guiding the horizontal flow of the eyes; and also to increase contrast and spacing between different letters for improved identification. San Serifs are used for emphases. Simplicity of the letter makes it more recognisable and even when blown up, it still maintain its general shape (UrbanFonts 2016). Interestingly, these subtle features make San serif fonts appropriate for the screen: Television, Computer monitors et cetera. Example of San Serif fonts are: Franklin Gothic, Calibri, Tahoma, Arial and Tiresias.

Harsen (2005) of BBC advised that "Gill" can be combined effectively with a body text face such as "Tiresias" for titles, headings and navigational elements while "Serif fonts" and light weight fonts should be avoided as they will not display well on television. With several rules researched to improve legibility on screen, Harsen (2005) further prescribed that no text should ever be smaller than 18 point (absolute minimum) in any circumstances, 24 point should be the general standard and no more than two typefaces should ever be used at once on screen. Light text on a dark background is slightly easier to read on screen, text on screen needs looser leading (i.e. great line spacing), a full screen of text should contain a rough maximum of 90 words and text should be broken into small chunks that can be read almost instantly (Harsen 2005). Appropriate tracking will definitely help to organise a screen filled with text. Generally, font weights sometimes possess some other attributes such as regular, condensed and expanded. Taking note of this, it is worthy of mentioning that condensed typefaces will not need much tracking compared to an 'expanded' character. Meanwhile, smaller font sizes will need more tracking than larger ones owing to the fact that they are relatively small.

\subsubsection{Features / Properties of a Typeface.}

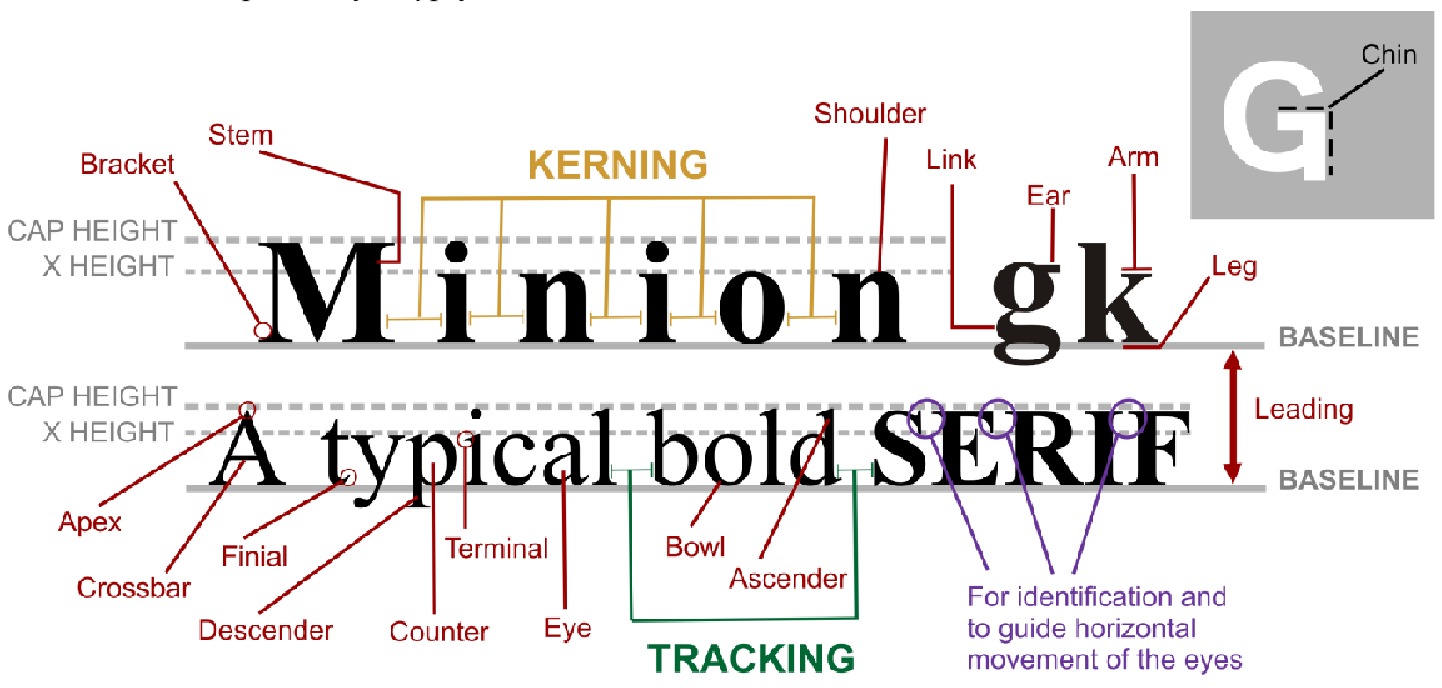

Figure 2, Anatomy of a type. (Source: D’Mediatix Creations, 2017)

The features of a type are unique with all letters and types are measured in points. From figure 2, font size is the distance that starts from the cap height (Apex of letter ' $A$ ') and extends beyond the baseline to the bottom of small letter ' $p$ ' that is having a descender. Letter ' $g$ ' and ' $y$ ' are other examples of letters with a descender. While descender is the extension (stem) protruding downward, ascender is specifically the part of small letters that extends upward beyond the x-height. Bowl is common with letters round, oval or sphere in shape; it is the enclosed portion that surrounds the counter. Counter on the other hand is the empty space inside a bowl. The link of letter ' $\mathrm{g}$ ' is the hook that joins its main parts. Kerning is the spacing between letters; tracking is the horizontal spacing in-between words. Leading is the spacing between lines or body of texts (Figure 2).

\subsection{Graphics and Images}

Television relies on images because of its visual modalities. Computer-Graphic Imaging (CGI) contributes immensely to content creation for TV; its use tends toward mostly high-end productions such as creation of 
photo-realistic 3D objects (Mckernan \& Cates, 2002). Graphics (digitally) is the representation of non-text information with the aid of primitive objects: lines, shapes, curves and illustrations such as cartoons, graphs, charts et cetera. Its application to Television further extend to: character generation (for text), retouching of still or motion pictures, and smooth integration into live-action digital video (Mckernan \& Cates, 2002). Computer generated imagery has also find its way into virtual set design as background effects for Television programmes. The technology of Chroma-key coupled with blue or green screen stage is adopted for the super-imposition of effects into the scene. Such scenery effect are characterised by three dimension (3D) models: images possessing the geometry of length, breadth and depth. Digital Tutors (2014) affirmed that whatever the geometry, three dimensions (3D) are created either by faces, points, edges or NURBs connected in a definite pattern with a flow around the 3D model - a description termed typology. Imaging is vital in television graphics which is a means to achieving visual solution for programming. Simultaneously, Television programmes, plainly visual contain multimedia of two and three dimensional graphic images (Afolabi, 2016). The appropriate and concise description of two dimensional arts is a vector based graphics and a bitmap image.

Vector graphics are designed electronically; using the computer system, but without third party facility such as scanner, camera et cetera (Mukhopadhyay \& Chattopadhyay, 2007). Vectors make use of points to define an image. They are created from a set of programmed algorithms; a geometrical formulas that specifies their directions, length and positions. Texts and shapes known as graphic primitives are examples of a vector (Vaughan 2011 \& Afolabi 2016). In titling, they help to generate a lasting movie logo that would be memorable; most especially when typefaces are stylised to capture specific features that link title directly to the content being produced (Braha, Y. \& Byrne, B., 2011). Manipulation of vector for multimedia content is a logical way of creating a brand to establish a programme identity. Digitally, vector graphic images are smaller in size and maintain their quality even when enlarged. In contrast, bitmaps are large in file size, needed in areas vector images cannot handle; as a result, they are real images (photographs) defined by a grid of pixels (Tilley, L. \& Park, D., 2005) - typically tiny dots of light which is the basic unit from which images on screens are made (Figure 3). Bitmap graphic images rely heavily on third party facilities to be captured and define its details which will either mar or make it. They are resolution dependant; camera for example will facilitate the pixels in bitmaps most importantly if the camera is high tech e.g. High Definition (HD) cameras. Resultantly, the more sophisticated the camera (in pixels), the higher the pixels of the image rendered (for a clear defined bitmap) and the heavier it (bitmap image) becomes in file size. As a plus, this is a good feature for television production where detailed graphic information matters. Besides, image size which proportionately means the height and width of an image, (measured in inches, centimetres, or pixels) is a factor that determines image quality.

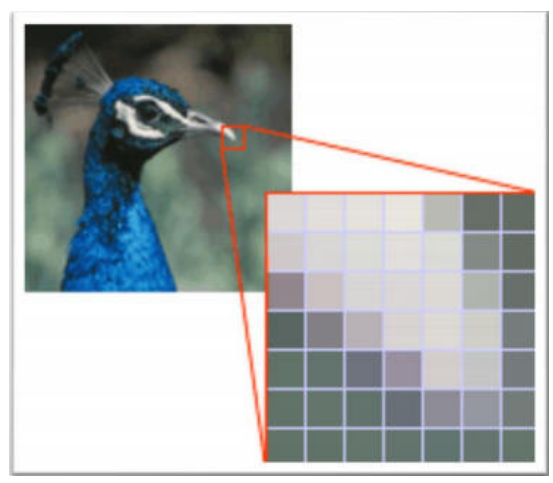

Figure 3, Pixels. (Source: FTMS College; Kuala Lumpur, Malaysia, 2016).

A subtle factor that determines and defines image quality also is colour depth. It is also known as bit depth: 1 bit, 4 bit and 8 bit etc. (Vaughan 2011). It is the series of colours available for pixels (Figure 4). 
iㅔ

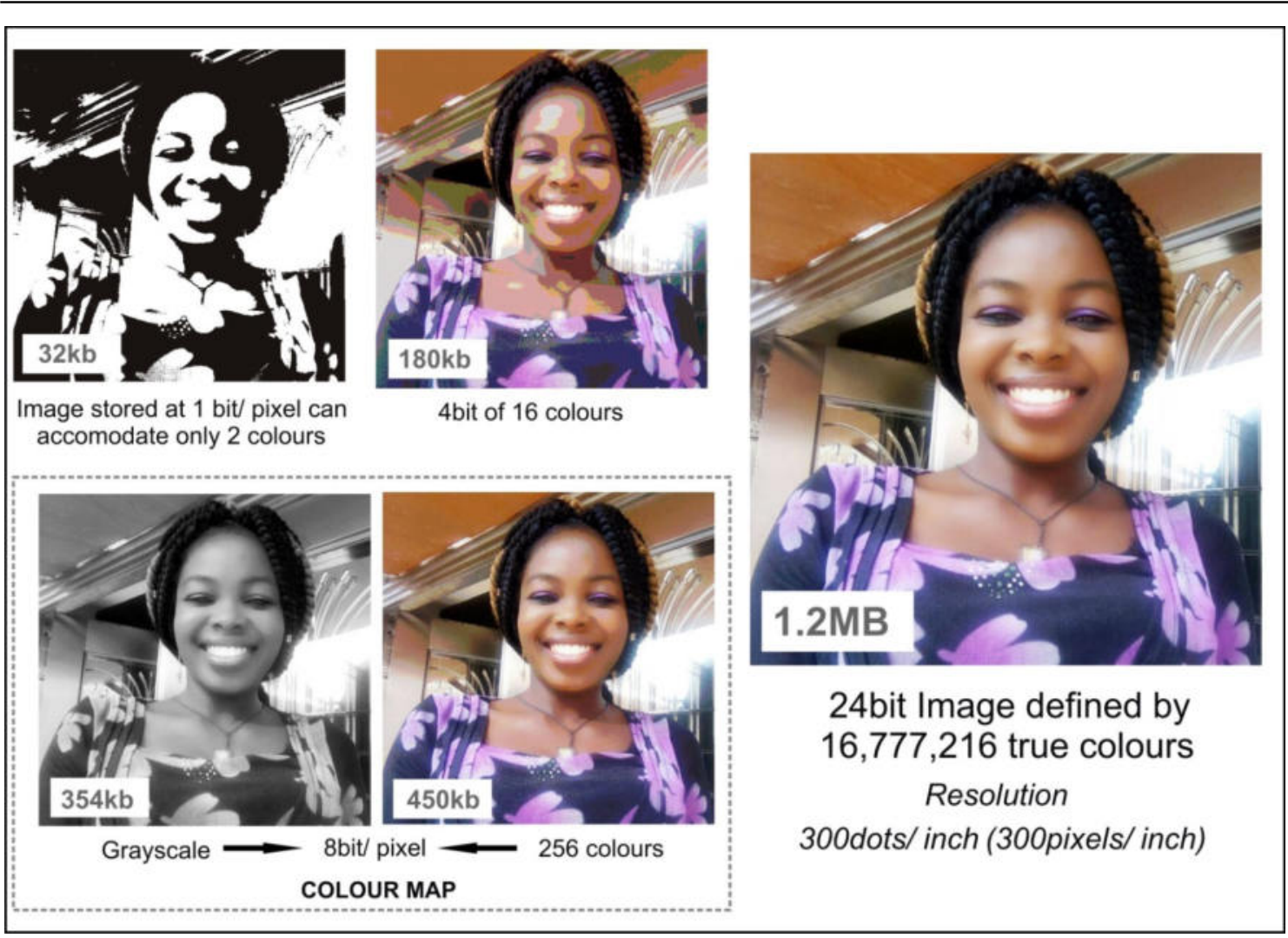

Figure 4, Colour depth with image size. (Source: Studio of D’Mediatix Creations, 2018)

A bitmap image with a very low colour depth may have just two different colours of black and white comparatively tapped from a master colour containing millions of colour palettes. Summarily, the lower the bit depth, the fewer the distribution of colours. At the same time, the higher the bit depth, the more colours that can be distributed to define an image. Another important factor that influences picture quality is 'resolution'. It is the sharpness of an image in terms of clarity as seen in Figure 4. Resolution, the number of pixels in an image equally contributed to the fine details of the 24bit image compared to others (Figure 4). The 24bit picture is rendered at dots/ inch (dpi) value of 300 while others vary between 100dpi to 190dpi. Photorealistic nature of bitmap solidifies its profile but the fluid nature of vector compliments its effectiveness when coupled together for visual design. The finely decorated graphic images and texts seen on television testify to this. Likewise, pictures of $3 \mathrm{~d}$ models such as texts composed in 3D (from vector to bitmap) are powerful assets for titling, and station identity/ logos. Practically, two and three dimensional designs go hand-in-hand. Afolabi (2016) posited that highly complex and geometric renderings (bitmaps) are generated by computer aided design (CAD) programs that traditionally use vector-drawn object systems. This is in line with the conclusion reached in a study by Mckernan \& Cates (2002) that such programs create objects (from vectors) with the aid of ' $X$ ', ' $Y$ ', and ' $Z$ ' coordinates taking cognisance of what their real position would be in real three-dimensional form. Bitmaps also known as raster graphics are stored with file extension which includes: .jpeg, .png, .tiff, .gif and .bmp while vectors' file extension formats are: .svg (Scalable Vector Graphics), and .ai (Adobe Illustrator).

\subsection{Audio - The Power of Sound}

Sound which is airborne vibrational waves perhaps, out of all the multimedia elements stimulates the senses the most. The vibrational waves remain audible to humans; modulated in any tone and in form of a sensible speech (whatever the language and mood used in its presentation). Mood in context refer to the system used to propagate the sound waves part of which encapsulates Television technology. Vaughan (2011) noted that sound brings pleasure and shock through music and special effects respectively; it also creates the atmosphere of a 'mood-setting background'. In their assertion, Braha \& Byrne (2011) summed up that music in a title sequence signals the introduction of the programme. 
Sounds in the definition of Centre for Disease Control and Prevention (2018) are known for frequencies (pitch) and intensity (loudness). Sound is also a terminology used in the analogue form while the digitized form is 'Audio' - music, speech, and structured audio: MIDI (Musical Instrument Digital Interface), AIFF et cetera (Afolabi 2016). Sound could be vowel or consonant produced by vibration in the varied movement of the vocal cord. Raw sound (vowel/ consonant), in the process of recording is heavier in size because of this, audio compression for Television production is very essential. Lee (2003) stated that substantial compression is needed to make transmission practical and it is done via Perceptual coding (based on the Science of Psychoacoustics) how people perceived sound. Biologically, sound according to Fischer (2008) is perceived through the ear; consisting of outer, inner and middle ear but also performs the multiple role of filtering, impedance matching, sound transmission over the air, conversion of sound waves to mechanical vibrations, and sound balancing, all of which actually lead to the hearing of the perceived encoded sound. In understanding how we hear Centre for Disease Control and Prevention (2018) and (Heather, 2014) posited that 20 to $20,000 \mathrm{~Hz}$ is the standard for human hearing range; and human ear is highly sensitive to sound that falls within 2,000 - 5,000 Hz. Likewise, important sounds heard in everyday activity is in range of frequencies between 250 to 6,000 Hz. Meanwhile, at $10,000 \mathrm{~Hz}$, the ear will not be able to pick sound clearly; similarly the ear cannot pick low frequency sounds very well such as sound at $30 \mathrm{~Hz}$. In day to day conversation, speech is a blend of low and high frequencies of both vowel and consonant sound (Centre for Disease Control and Prevention 2018). As stated before, raw sounds are heavy in file size; a complete fix is compression. Sound can be compressed with different encoding technologies; notable among the perceptual coding is the MP3. It is a format developed by the Moving Picture Experts Group - 'MPEG' (Vaughan, 2011).

MPEG is a standard embraced for transmission of sound in Television Stations; simultaneously with MPEG audio, AC-3 audio (Dolby digital audio standard) is also well accepted (Fischer 2008). Apart from using Dolby digital audio standard in digital terrestrial television broadcasting, the AC-3 audio developed by Dolby Labs in the USA is highly patronised by the film industry for movies and DVD technologies. Quality wise, Fischer (2008), argued that there is no difference between MPEG audio and Dolby digital as both are having improved codecs. Presently, Dolby AC-3 audio transforms coding based on Modified Discrete Cosine Transform (MDCT) using five in one audio channels (subwoofer system inclusive). Bitrate standardised to $128 \mathrm{~kb} / \mathrm{s}$ per channel. MPEG, in like manner transmuted to MPEG-2 (using AAC i.e. Advanced Audio Coding) and MPEG-4 (AAC and AAC+) (Fischer, 2008). However, apart from audio codecs, appropriate manipulation of variables such as sampling rate (in kilohertz) and bitrate (in kilobyte per seconds) would practically affect the output quality of a digital audio. The higher the bitrate of an audio file, the greater the dynamic range with better sound output compared to the same audio file compressed at a lower bitrate. The clear sound, Christensson (2006) explained is as a result of more bits stored to represent the audio data (for each second) to be played back. Convincingly, a higher bit depth produces a greater resolution (dynamic range) with respect to amplitude of the waves while a higher bitrate results in a greater resolution with respect to frequencies (Christensson 2006). Moreover, the sound profile of an audio as regards quality is based on the encoding (via recording/ compression) and not the end users' gadget for playback. Hence, digital audio is device independent (Vaughan 2011). Sound is a sensitive element of multimedia but in terms of production, people mostly do not give attention to it (compare to video) except the audio output is so bad that it is conspicuous. Whereas, sound distinctively accompanies video in multimedia communication; more reason television broadcasting is audio-visual. On account of this, Tully (2002) clarified that audio is more than half the picture; while it is illogical to underestimate the power of the spoken word i.e. sound (Nayyar 2007).

\subsection{Video and Animation}

The power of motion synonymously describes animation and video (Afolabi, 2016). Motion picture and Video, most especially are the elements of Multimedia that are noticeably common. These elements tagged 'continuous media' is an interesting and unavoidable aspect of multimedia. It improves understanding and brings reality to the linear nature of Television (Afolabi, 2016). Besides, animation is witnessed in titling. A title sequence is a means to express the power of motion graphics and a creative approach to open a TV show or a movie.

Animation is created from drawn still pictures (Figure 5). Video as well is the technology of processing a sequence of still images to depict scenes in motion; a photo-electric transducer is employed. Contrastingly, while video takes continuous motion and breaks it into discrete frames; animation starts with independent pictures, binds them together to form an illusion of continuous motion (Lee 2003) as reflected in Figure 5. The rapid display of a sequence of images (2D or $3 \mathrm{D}$ models) positioned in order to create an illusion of movement due to 
the phenomenon of 'persistence of vision' by the human eyes as explained by Vaughan (2011) is Animation. To affirm this view, Afolabi (2016) supported that the human eye has the property that when an image appears on the retina, the picture is retained for some number of milliseconds before decaying. Logically, sequence of image drawn line by line at sixty (60) images per second, will remain extremely unobvious to the eye as discrete image when viewed. Television broadcasting and Video technology key into this principle for the production of moving pictures (Afolabi 2016). Meanwhile, in the case of television, videos can generate frames or pictures every second. Some scholars regard raw video as series of single images; typically 25, 30 or 60 frames per seconds (fps). It is the rate of replacement (in terms of speed) of each frame one after the other that creates a smooth blend of motion picture (Vaughan 2011).

A Video file is always very heavy in size therefore its compression (in lossy and lossless format) is encouraged before transmission. Some digital media files for animation and video are:

i. Animation: 3d Studio Max (.max), QuickTime (.qt), Adobe Flash (.fla and .swf) etc.

ii. Video: RealMedia (.rm), Windows Media Video (.wmv), Audio-Video Interleave (.avi),

MPEG (.mp4), Matroska (.mkv), QuickTime MOVie (.mov) etc.

The operational mode of a video in the discussion of Lee (2003) is that the reflection of light (from an object) passes through the lens of a camera as the camera scans three electron beam (of red, green and blue) rapidly across and slowly down the object. The camera (as it engages in uniform movement) records the light intensity. The scanning process upon completion creates a frame, each beam then retraces again to display the object (now an image) in true colours. The 'intensity' in real time is broadcast; the receivers repeat the scanning process to rebuild the image for visibility and accessibility (Lee, 2003).

Digitally, an image is produced in video camera with the aid of an integrated sensor called charge-coupled device (Vaughan 2011) and does not engage in scanning system. The technology behind it is that the camera records the available light when focused on an object then exposes the charge-coupled device (CCD) to the captured light to build up an electrical charge. Digital Video Cameras make use of pixels; grid of dots. A mini processor in the camera compresses and interpolates the data from the pixels to create the colours; at this point, the image is accessible (Reddy 2009). Rouse (2010) emphasis is that CCD in a digital camera ensures quality output; some camcorders even produce images with over a million pixels - an improvement over the analogue technology. Video and animation share common attributes, but video is the most engaging of media elements; powerful tool and an excellent method for delivering multimedia to an audience raised on the Television. Significantly, before video became widely patronised, means of active actions in multimedia designs was 'animation' (Vaughan 2011).

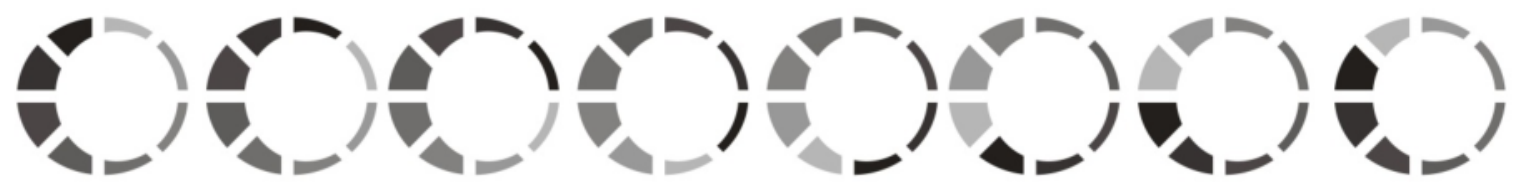

Figure 5, Still Pictures: illusion of continuous motion (Animation).

Source: Author's Collection, 2017

Broadcast standards also aid the display of visuals in television programming but this study will not delve extensively into this aspect since it is not the main focus of the research. Known standards by Vaughan (2011) are: NTSC (National Television Standards Committee), PAL (Phase Alternating Line), and SECAM (Séquentiel couleur à mémoire or Sequential Colour with Memory). They are characterised by a scanning system and a welldefined aspect ratio. Digitisation bred high definition television; it produces sharper images by roughly doubling the number of scan lines. High definition is produced in 16:9 aspect ratio and with this configuration, cinematic movies are possible (Jinxiang \& Waddel, 2005). Television programmes (live or recorded) can be rendered in full high definition; video that will be produced will have dimensions of 1920 X 1080 (in pixels) - a standard for high definition.

\section{Television Programme: 'Bond of Multimedia Elements'}

Television appeals to all ages because of its 'programming' involving all the media elements in composition. From the network schedule of Nigerian Television Authority (NTA) - third quarter, 2018, television programmes are categorised within a specified genre: News and Current Affairs, Documentaries, Entertainment programmes: Musicals, Talk shows, sports, Drama and Soap Opera, Educative and Religious programmes, local 
and international flavours etc. The television is able to fulfil its primary role through the News and Current Affairs, assisting in the interpretation and prescription of news. Sambe (2004) in his explanation stressed that the filtering of news help to prevent over stimulation and simultaneously over mobilisation of the society. Reports from local scene, regional, national, and international events are all features of news programme. It is common these days that weather reports are embedded in the news programme. A good number of programmes are designed for analysis and interpretation. Topics are open to discussions via talk shows, panel shows, consumer affairs or reviews, news magazines and documentaries etc. This type of programmes could either serve entertainment purposes or formally to inform the society. On the other hand, entertainment programmes such as drama, sports, musicals, and reality shows are simply to create a calm atmosphere, make people relax and laugh. Reality TV shows are unscripted and acted humours; in which real happenings are recorded but sometimes directed therefore takes the shape of soap opera. As a result, reality shows are also known as either “docudramas" or “docusoaps" (Canadian Radio-television and Telecommunications Commission, 2018).

It is worthy to note that leisure programmes can serve dual purpose of entertainment and education. People are educated using humorous contents: cartoon is a simple example. They are drawn, animated in 3D for real time lifelike experience. This form of edutainment is gaining more attention in the twenty-first century. Long-form documentary are specifically created to inform but may also fall within the category of an edutainment programme. Documentaries of this type give room for an in-depth critical analysis of a special topic. Meanwhile education programmes are strictly formal presentations covering body of knowledge: Maths, Physics, Vocational Studies, quizzes, puzzles and educative game shows. Educative programmes provide viewers with meaningful set of skills. Ones that are packaged in line with school subjects (to serve as supplements) are usually designed around school curriculum. Educative multimedia contents can deal with formal, non-formal and informal learning which will make people to be conscious of their environment for societal and national development. Religious programmes make man to be aware of his spirit being and same time is alert to the Supreme Being: witnessed via church gatherings, open plaza crusades, evangelism talk shows and group discussions. Local contents can take form of any genre specified above; the main aim is to present stuffs and varieties in a local manner. By definition varieties in programming is a show consisting series of short unrelated performances; a programme of mixed characters: poem recitation, magic, choreography, acrobatic displays, vox pop, comedy sketches etc. (Bravolol 2018).

Programmes can also be aired in form of PSAs (Public Service Announcements), Commercials or Promotional Adverts. While PSAs (Public Service Announcements) serve the purpose of announcement to the general public, Commercials are adverts meant to campaign (either to a targeted audience or general public) about a particular products and services. Capital aim to drive sales and position the brand in better perspectives. The impact of multimedia elements to build up contents is of high relevance. A typical example is derived from a television programme of NTA (Nigerian Television Authority), Channel 12, Abeokuta, titled: "Healthy Living". The health programme draws its strength from visual illustrations: graphics, images and texts, etc. mixed and incorporated into the programme for clarity of information (NTA, Abeokuta 2018). Similarly, commercials are known to be short, passing across promotional messages within the limited time frame - a feature made possible due to the possibility of blending multimedia elements.

\section{Linear Nature of Multimedia and Television Programming in Nigeria}

Television broadcasting is the transmission of multimedia contents (i.e. programmes); from a base station, technologically with the aid of electromagnetic spectrums and air waves to a large and widely scattered audience. Encoded signals (programmes) are sent to reception devices such as television sets in homes or relay stations such as those used by cable television service providers (Antonoff, 2008). Television programmes contains multimedia of two and three-dimensional graphics. Text, images and photographs are two dimensional while video and animated films are projected in three-dimensional mode. Products of multimedia can be used in diverse ways while the media elements are integrated in different formats. Variation in the methods and formats make multimedia dynamic in nature, experienced both linearly and non-linearly (Afolabi 2016). Multimedia presentation that is linear in nature accommodates any multimedia element but the output most times lacks interactivity thus 'passive' (Vaughan 2011). Attributes of linearity establish that a multimedia project will start with a defined beginning and stop at a timed end with nothing disrupting the flow of the presentation. The page by page approach lacks menus, buttons or navigational links - the main features that command interactivity. Television accommodates this technology thus makes it (TV) a linear platform. 
The linear nature of TV is exemplified in the 'frame-to-frame' display of multimedia contents; principally a way of arresting attention. In justifying this standard, Afolabi (2016) noted that when the goal is simply to entertain, gain knowledge about a subject or become familiar with other's viewpoints, a linear format is purposely preferred because it allows for greater immersion in the experience. The process from gathering information and packaging to its dissemination equally takes linear mode. From the explanation of Campbell, Martin \& Fabos (2012), on the linear model of mass communication, logically, it can be inferred that Producers (Senders) transmit programmes- sounds and visuals (multimedia contents) through television, or the Internet (media channels) to a large heterogeneous audience (viewers and consumers). In the process, executive producers and other media stakeholders (gatekeepers) function as content filters and censor agents. They make decisions and finalise on programmes to be produced and aired, for the appropriate viewers. In return, the linear system gives room for consumers to provide feedback (optional though) to the appropriate quarter via: talk shows, letters, phone calls, online platforms: social media, email, and filling of web forms etc. As a result, it is easy to conclude that in the principles and practice of mass media, Television (a platform for multimedia) is also assessable on the basis of linearity. The linear process looks fluid but considering the lamentation of Campbell, Martin \& Fabos (2012), in reality media messages do not truly move smoothly as perceived. Information is more likely to get mixed up in the rigorous process of communication activities. Practically, the information encoded in visual form ends with the receiver (audience) but, the encoder has little or no control over how the multimedia messages are decoded: whether accepted, misinterpreted or ignored completely Campbell, Martin \& Fabos (2012).

It is worthy of mentioning that, when multimedia is rich in interactivity, it is said to be 'non-linear' (Vaughan 2011). Though it is observed that Television is basically of linear mode but advancement has brought about interactivity with the aid of remote controls to scan through Electronic Programme Guide (EPG) in digital broadcasting. Additionally, interactivity can surface via phone-in as the presenter is anchoring the programme. A dedicated number would have been released to the viewers. These levels of interactivity may not be intense but to an extent, it breaks the mundane way of accessing content of the screen - a linear platform. Interactivity (core feature of nonlinear platform) is in a steady pace finding its way into linear television to realign it with the evolving technology. Cesar and Chorianopoulos (2009) in their research discussed the state-of-the-art in interactive television user developments. They identified three basic concepts of content editing, sharing, and control - an evolutionary step over the established linear pattern of produce, distribute and consume, which is the usual mode of operation in television. They identified if well considered, that those three areas will aid development of quality multimedia products for TV; as content creation remains key in the future of television. This is a clear signal that television is transcending beyond the usual one way passive approach. While contents are scheduled for broadcasting in linear TV, in which timing cannot be changed by media consumers, digital technology introduces Personal Video Recorder (PVR) and on-demand strategy - a typical nonlinear technique to enhance mode of content delivery. Viewers can record a programme (into the hard disk of a setup box) for later viewing (known as Time Shifted Viewing). Broadcasters as well can provide contents to be readily available for on-demand services. The nonlinear mode of operation is being influenced by the availability of internet, satellite and dynamic smart devices that support accessing multimedia contents at any point in time, within any geographical location. In a simultaneous manner, as TV maintains its linearity via the introduction of high definition programmes (for relevance), by year 2020 there will be more watching of those programmes (at least $50 \%$ ) in a nonlinear mode (IHS Inc. 2011 \& CBS TV Network, 2016). Television programming in Nigeria is relatively guided by laid down standards of the National Broadcasting Corporation (NBC). Virtually all stations are currently abiding with the rule of digitization to aid smooth transition - analogue switch off and digital switch over. At the moment, as reported by Olowu (2018) "funding has been a long standing demon" hindering the realisation of complete digital switch over. A technology which will eventually strengthen creation of TV programmes in Nigeria. Apart from funding, what other efforts need to be geared up for quality content production, most importantly from the perspective of multimedia design for television?

In a survey carried out by Afolabi (2016) in Lagos State, Nigeria, out of the total number of sixty-eight (68) questionnaires administered to Multimedia Editors of television programmes in broadcasting stations in Lagos state, sixty-two (62) representing $91.2 \%$ of the total number administered were returned and used for analysis. Census was used as a sampling technique since the population was finite. Lagos was chosen as area of concentration because it is the state with the largest number of television stations in Nigeria. All the TV stations equally have their headquarters situated in the centre of commerce which is Lagos State. The study reveals that out of all the multimedia elements used in programme production, graphic and images, audio, video and text 
constituted a greater portion with a mean score of 4.97, 4.97, 4.94 and 4.81 respectively while animation is the least used with a mean score of 3.23 (Table 4). Meanwhile the Editors 52 (83.9\%) strongly agreed and 10 $(16.1 \%)$ also agreed that a mix of multimedia elements bring life to conceived ideas and stories and it visually communicate better. None of the Programme Editors disagreed nor were undecided (Table 3). Inferably, they were convinced of the importance of integrating text, graphics, images, audio, video and animation; yet those multimedia elements are used unevenly as witnessed in Table 4. The Editors made it known (through discussions) that, though, "animation" is a needed element of multimedia but it is less used because it is exceptionally difficult to create. Observing further on the features of multimedia, the Multimedia Programme Editors 49 (79\%) strongly agreed, 19.4\% agreed that Images, Text, Sound, Graphics, Animation and Video are elements of multimedia (for programme production). An Editor (1.6\%) remained undecided (Table 3). The high percentages solidify the established opinion by several scholars that programmes are the fuels that drive television broadcasting and what make up the programmes are the multimedia elements.

Table 3: Features of Multimedia

\begin{tabular}{|c|c|c|c|c|c|c|}
\hline Variable & Response & Freq. & \%tage & Mean & Std. Dev. & Remark \\
\hline \multirow{6}{*}{$\begin{array}{l}\text { Images, Text, Sound, } \\
\text { Graphics, Animation and } \\
\text { Video are Elements of } \\
\text { multimedia in programme } \\
\text { production for Television }\end{array}$} & Strongly Agree & 49 & $79.0 \%$ & \multirow{5}{*}{4.76} & \multirow{5}{*}{.534} & \multirow{5}{*}{ Valid } \\
\hline & Agree & 12 & $19.4 \%$ & & & \\
\hline & Undecided & 1 & $1.6 \%$ & & & \\
\hline & Disagree & \multirow{2}{*}{\multicolumn{2}{|c|}{-}} & & & \\
\hline & Strongly Disagree & & & & & \\
\hline & Total & 62 & $100 \%$ & & & \\
\hline \multirow{6}{*}{$\begin{array}{l}\text { A mix of multimedia } \\
\text { elements brings life to the } \\
\text { conceived idea/ story and } \\
\text { visually communicates } \\
\text { better. }\end{array}$} & Strongly Agree & 52 & $83.9 \%$ & \multirow{5}{*}{4.84} & \multirow{5}{*}{.371} & \multirow{5}{*}{ Valid } \\
\hline & Agree & 10 & $16.1 \%$ & & & \\
\hline & Undecided & - & & & & \\
\hline & Disagree & - & & & & \\
\hline & Strongly Disagree & - & & & & \\
\hline & Total & 62 & $100 \%$ & & & \\
\hline
\end{tabular}

Source: Field Work, Afolabi (2016).

Table 4: Multimedia Elements widely used in packaging programmes for TV

\begin{tabular}{|c|c|c|c|c|c|c|}
\hline Variable & Response & Frequency & Percentage & Mean & Std. Dev. & Remark \\
\hline \multirow{6}{*}{ Text } & Very Often & 50 & $80.6 \%$ & \multirow{5}{*}{4.81} & \multirow{5}{*}{.398} & \multirow{5}{*}{ Valid } \\
\hline & Often & 12 & $19.4 \%$ & & & \\
\hline & Undecided & - & - & & & \\
\hline & Not Often & - & & & & \\
\hline & Not Very Often & - & & & & \\
\hline & Total & 62 & $100 \%$ & & & \\
\hline \multirow{6}{*}{ Graphic/ Images } & Very Often & 60 & $96.8 \%$ & \multirow{5}{*}{4.97} & \multirow{5}{*}{.178} & \multirow{5}{*}{ Valid } \\
\hline & Often & 2 & $3.2 \%$ & & & \\
\hline & Undecided & - & - & & & \\
\hline & Not Often & - & & & & \\
\hline & Not Very Often & - & & & & \\
\hline & Total & 62 & $100 \%$ & & & \\
\hline \multirow{8}{*}{ Animation } & Very Often & 5 & $8.1 \%$ & \multirow{5}{*}{3.23} & \multirow{5}{*}{1.093} & \multirow{5}{*}{ Valid } \\
\hline & Often & 30 & $48.4 \%$ & & & \\
\hline & Undecided & 1 & $1.6 \%$ & & & \\
\hline & Not Often & 26 & $41.9 \%$ & & & \\
\hline & Not Very Often & - & & & & \\
\hline & Total & 62 & $100 \%$ & & & \\
\hline & Very Often & 60 & $96.8 \%$ & & & \\
\hline & Often & 2 & $3.2 \%$ & & & \\
\hline
\end{tabular}




\begin{tabular}{|c|c|c|c|c|c|c|}
\hline \multirow[t]{4}{*}{ Audio (Sound) } & Undecided & \multirow{3}{*}{$\begin{array}{l}- \\
- \\
-\end{array}$} & \multirow[t]{3}{*}{ - } & \multirow[t]{3}{*}{4.97} & \multirow[t]{3}{*}{.178} & \multirow[t]{3}{*}{ Valid } \\
\hline & Not Often & & & & & \\
\hline & Not Very Often & & & & & \\
\hline & Total & 62 & $100 \%$ & & & \\
\hline \multirow{6}{*}{ Video } & Very Often & 58 & $93.5 \%$ & \multirow{5}{*}{4.94} & \multirow{5}{*}{.248} & \multirow{5}{*}{ Valid } \\
\hline & Often & 4 & $6.5 \%$ & & & \\
\hline & Undecided & - & - & & & \\
\hline & Not Often & - & & & & \\
\hline & Strongly Disagree & - & & & & \\
\hline & Total & 62 & $100 \%$ & & & \\
\hline
\end{tabular}

Source: Field Work, Afolabi (2016).

Considering the perception of respondents on problems of multimedia production for television (Table 5); using non-design experts to package Multimedia contents which demean quality of production ranked highest by the Programme Editors (with a mean of 4.76) despite lack of funds being a serious issue as well (Table 5). Thus using non-design experts to package multimedia contents (which demean quality of production) remains the greatest challenge. This glaringly, further account for the uneven use of the media elements (for multimedia design); to package contents for Television (as evident in Table 4). In addition, greater percentage (95.1\%) of the respondents agreed that dearth of funds is a serious problem that affects quality of multimedia production - mean score 4.44 (Table 5). Perfectly, it corroborates the observation of Olowu (2018) that "funding has been a long standing demon" hindering the realisation of complete digital switch over in Nigeria.

Table 5: Problems of Multimedia production

\begin{tabular}{|c|c|c|c|c|c|c|}
\hline Variable & Response & Freq. & \%tage & Mean & Std. Dev. & Remark \\
\hline \multirow{6}{*}{$\begin{array}{l}\text { Dearth of funds is a serious } \\
\text { problem that affects quality } \\
\text { of multimedia production. }\end{array}$} & Strongly Agree & 32 & $51.6 \%$ & \multirow{5}{*}{4.44} & \multirow{5}{*}{.671} & \multirow{5}{*}{ Valid } \\
\hline & Agree & 27 & $43.5 \%$ & & & \\
\hline & Undecided & 1 & $1.6 \%$ & & & \\
\hline & Disagree & 2 & $3.3 \%$ & & & \\
\hline & Strongly Disagree & - & & & & \\
\hline & Total & 62 & $100 \%$ & & & \\
\hline \multirow{6}{*}{$\begin{array}{l}\text { Using non-design experts to } \\
\text { package Multimedia } \\
\text { contents which demeans } \\
\text { quality of production. }\end{array}$} & Strongly Agree & 50 & $80.6 \%$ & \multirow{5}{*}{4.76} & \multirow{5}{*}{.564} & \multirow{5}{*}{ Valid } \\
\hline & Agree & 10 & $16.2 \%$ & & & \\
\hline & Undecided & 1 & $1.6 \%$ & & & \\
\hline & Disagree & 1 & $1.6 \%$ & & & \\
\hline & Strongly Disagree & - & & & & \\
\hline & Total & 62 & $100 \%$ & & & \\
\hline
\end{tabular}

Source: Field Work, Afolabi (2016).

\section{Conclusion and Recommendation}

The study among other areas of relevance has been able to identify multimedia elements as a means of visual communication, and inevitable features of television programmes. The point of focus is the integration and convergence of the five media elements in which the result of this research reveals the preference of other multimedia elements over animations. The result further clarifies that Multimedia Programme Editors maintained the use of non-design experts in packaging programmes has negative impact on production quality. Therefore, it is expected that research like this will help in the improvement of multimedia design for the screen, contribute positively to the practise and also to the body of knowledge. However, improvement in multimedia design can only be recorded when there is proper funding for facilities and frequent training of Editors. Most importantly, multimedia specialists should be employed to package television programmes such a person will be well versed in Visual design, Visual perception and Cognitive psychology. Multimedia designers should make efforts to utilise multimedia building blocks creatively; that there will not be disparity in its usage to the extent of marring a worthy production. In a bid to revive dwindling cultural heritage, synergy between Visual designers and Media Industry is encouraged to foster more projection of quality local contents, using the multimedia elements on the platform of television. 


\section{References}

Afolabi, B. F. (2016). Investigating the Use of Multimedia Elements for Visual Communication in Television Stations in Lagos State, Nigeria. Unpublished M.Tech Thesis, Akure: Department of Industrial Design, Federal University of Technology.

Akpan, E. (1990). Beyond Message Transmission: A Comparative Approach to TV Production. Nigerian Journal of Mass Communication. Vol. 1, No. 1.

Antonoff, M. (2008). "Television." Microsoft ${ }^{\circledR}$ Encarta ${ }^{\circledR} 2009$ [DVD]. Redmond, WA: Microsoft Corporation.

Braha, Y. \& Byrne, B. (2011). Creative Motion Graphic Titling For Film, Video and the Web. Burlington, MA: Focal Press

Bravolol Inc. (2018): Variety Show URL: http://www.bravolol.com Accessed on Nov. 14th, 2018

Campbell, R., Martin, C., \& Fabos, B. (2012). Media and Culture: An Introduction to Mass Communication. $8^{\text {th }}$ Ed. Boston, MA: Bedford/ St. Martin's

Canadian Radio-television \& Telecommunications Commission (2018). Television Program Categories. URL: https://crtc.gc.ca/canrec/eng/tvcat.htm Accessed on November 24th, 2018

CBS Television Network (2016). "CBS Sees 50 Per cent Non-Linear TV Future" URL: https://www.warc.com/LatestNews/News/CBS_sees_50_nonlinear_TV_future.news?ID=35223

Accessed on 1st October, 2017

Centre for Disease Control and Prevention (2018). Hearing Loss in Children. URL: https://www.cdc.gov/ncbddd/hearingloss/sound.html Accessed on August 9th, 2018

Cesar, P. \& Chorianopoulos K. (2009). The Evolution of TV Systems, Content, and Users Toward Interactivity. Foundations and Trends in Human-Computer Interaction. 2 (4): 279-374

Christensson, P. (2006). Bitrate Definition. URL: https://techterms.com/definition/bitrate Accessed 20/11/18 Daramola, I. (2003). Introduction to Mass Communication. Lagos: Rothan Press.

Digital Tutors (2014). Modelling Terms. URL: www.digitaltutors.com Accessed 02/11/2017

Fischer, W. (2008). Digital Video and Audio Broadcasting Technology. ${ }^{\text {nd }}$ Ed. Heidelberg, Germany: Springer Fowler, O. M (2000). Innovative Design Principles. Newbury: Aalborg Books International Gibson, M. (1979). Editing in the Electronic Era. Iowa: Iowa State University Press.

Harsen, V. (2005). Interactive Television Design (BBC). URL: http://www.bbc.co.uk/interactivetelevision forum/2012/12/12 itvd.pdf. Accessed on 12 $2^{\text {th }}$ Dec. 2012.

Heather, S. (2014). The Science of Hearing. URL: http://www.simonheather.co.uk/pages/articles/science hearing.pdf Accessed on Sept. 25th, 2018

IHS Inc. (2011). Broadcasters Have No Cause for Panic Over Rise of Nonlinear TV. URL: https://technology.ihs.com/389350/broadcasters-have-no-cause-for-panic-over-rise-of-nonlinear-tv Accessed on 15th May, 2018

Jinxiang Yu \& Waddel, D. (2005). Multimedia Effectiveness, Developing a Strategy. Proceedings of the 2005 IEEE Frontiers in Education Conference, Savannah: GA. Retrieved October 9th, 2018 from http://fie.engrng.pitt.edu/file2005/papers/1564.pdf

Lee, P. H (2003). Visual Communication and Television Design. Cambridge; MIT Press

Mckernan, B. \& Cates, R. (2002). CGI and Digital Content Creation. In Rice, J. \& McKernan, B. (Ed.), Creating Digital Content: Video Production for Web, Broadcast \& Cinema. New York: McGraw-Hill Companies, Inc.

Mukhopadhyay, A \& Chattopadhyay, A. (2007). Introduction to Computer Graphics and Multimedia. Kolkatta: Vikas Publishing House.

Nayyar, D. (2007). Modern Mass Communication (Concepts and Processes). Jaipur: Oxford Book Company

NTA 12, Abeokuta (2018). About Us: Мenu URL: https://www.ntaabeokuta.com/about Accessed Dec. 31st, 2018

NTA (2018). Programme Schedule: $3^{\text {rd }}$ Quarter 2018. URL: http://www.nta.ng/program-schedule Accessed September 9th, 2018

Olowu, D. (2018). Nigeria's digital switchover: Let us move forward URL: https://www.vanguardngr.com/2018/11/nigerias-digital-switchover-let-us-move-forward/ Accessed 29/12/ 2018

Quinn, S. (2005). Digital Sub Editing and Design. Oxford: Focal Press

Reddy, S. (2009). CCD (Charge Coupled Device). URL: https://www.slideshare.net/mobile/SagarRed/ccdcharge-coupled-device/ Accessed 09/08/2018

Rouse, M. (2010). Charge-Coupled Device (CCD). URL: https://searchstorage.techtarget.com/definition/chargecoupled-device Accessed 23/10/2018 
Sambe, J. (2004). Introduction to Mass Communication. Lecture Monograph. Lagos: Nigeria. Tilley, L. \& Park, D. (2005): Computing Studies: Multimedia Application. Scotland: LT Scotland Tully, T. (2002). Audio: More Than Half the Picture. In Rice, J. \& McKernan, B. (Ed.), Creating Digital Content: Video Production for Web, Broadcast \& Cinema. New York: McGraw-Hill Companies, Inc. UrbanFonts (2016). Serif Versus Sans: The Final Battle. URL: www.urbanfonts.com Accessed 07/07/2017 Vaughan, T. (2011). Multimedia: Making It Work. $8^{\text {th }}$ Ed. New York: McGraw-Hill Companies. 